\title{
Issues Worthy of Our Attention: Perspectives on Current Business Ethics Issues from Accounting, Finance, Management and Marketing Scholars
}

What are the contemporary business ethics issues that the business ethics field needs to address? Noted scholars answered that question at the University of Notre Dame's sixth annual "Ethical Dimensions in Business: Reflections from the Business Academic Community" conference and those thoughts are captured in the collection of papers in this issue. Drawn from the accounting, finance, management, and marketing fields, these scholars were asked to identify ethical issues within their domain of study. Key to this identification is that they are not "ethicists" by training but rather are noted academics within their discipline. As will be evident as you read their papers, the discussions provide a fresh perspective on the issues that are in critical need of attention, discussion, and future research.

In the "Distribution of IPO Holdings across Institutional Funds," Johnson and Marietta-Westberg examine initial public offerings (IPO) and their distribution across a sample of fund families. In doing so, several important ethical issues are identified, including the practice of holding IPOs outside of the fund's objective, a practice that could be argued to misleading to investors who rely on the stated objectives of the fund to achieve their investment objectives, such as balancing their account. The implications of these issues are important not only for investors but also for identifying improvements in the practices at investment and non-investment banks.

Forster, Loughran, and McDonald, in "Commonalities in Codes of Ethics," highlight the similarities in ethics codes between firms. The similarities noted raise concern over the potential impact that such "rubber stamped" value statements have, if in fact, they are not developed within a specific organizational culture but rather are "copied" from firm to firm. In addition, the possibility that regulation is at least partly responsible for these similarities suggests that we need to consider the implications of "mandating" ethics to organizations.

In "The Use of Scents to Influence Consumers: The Sense of Using Scents to Make Scents," Bradford and Desrochers analyze the ethical issues associated with scent marketing. Such a practice, which involves the use of scents to influence consumers, is argued to raise troubling ethical issues in the marketing field because of the subliminal, "unstoppable," and unobserved nature of the sense of smell. As discussed, these issues are not limited to retailers but need to be addressed by researchers as well.

The connection between religiosity and ethical judgments is explored in Vitell's, "The Role of Religiosity in Marketing and Consumer Ethics Research." Vitell provides an overview of the incorporation of religiosity in ethics research in the marketing field, noting the growing influence of the religiosity construct and its relationship to ethical judgments. Vitell's review suggests that additional research is needed in this area, particularly in understanding the relationships between religiosity and moral philosophy, moral awareness, and moral behavior.

In "Protect My Privacy or Support the Common Good? The Ethical Dilemma of the Opt-in versus Optout Decision related to Electronic Health Information Exchanges," Angst raises the very timely topic of electronic health information exchange and the important ethical issues it raises. Argued to provide a common good, an electronic health information 
database is also fraught with ethical issues, including concerns over privacy and trust. Angst's work informs many relevant scholarly areas - including social dilemmas and stakeholder theory - and, in doing so, identifies a fruitful area for future research.

Gaa discusses the issue of strategic financial reporting in "Corporate Governance and the Responsibility of the Board of Directors." As the discussion identifies, the process of strategic financial reporting is one that is currently without accountability, and as such, there is no clear ownership of the complicated issues that arise. Without defined responsibilities for this issue, which Gaa argues should rest in the hand of the Board of Directors, issues of moral intention and egocentric biases become relevant topics for future study.

A comprehensive overview of bribery and corruption is provided by Cleveland, Favo, Frecka, and Owens in "Trends in the International Fight Against Bribery and Corruption." In addition to providing an informed review of bribery and corruption and a typology that identifies the types of mechanisms that are used in the fight against bribery, the ethicality of such acts are discussed from an equity, efficiency, and fairness perspective. In and of itself, the review provides a very solid template upon which additional research can be solidly grounded; further, the authors identify several pressing concerns that need further study, including the impact of bribery on corporate reputation and the extent to which antibribery mechanisms will be effective in other types of corporate actions such as corporate stewardship.
In sum, these papers offer a very solid grounding upon which future research can flourish. The papers by Johnson and Marietta-Westberg and Forster et al. offer empirical research on the organizational practices of holding funds outside their classification and the "copying" of ethics codes, respectively, raising both immediate practical implications for the business world and scholarly issues for future study. The articles by Bradford and Desrochers and Angst each identify emerging ethical issues in the business world - i.e., scent marketing and electronic health information exchange - highlighting the importance of studying these "new" issues from a scholarly perspective. Yet other articles, such as those offered by Gaa, Cleveland et al., and Vitell, offer solid reviews of critical issues in business ethics research, reviews that can serve as informed and comprehensive platforms upon which future research in these areas can be conducted.

These authors were asked to take a look at their business discipline and identify and discuss the ethical issues that were in need of future research. Each paper is unique in the perspective and issues that are tackled. Yet, despite different perspectives, these articles all share a commonality in providing a useful and exciting roadmap upon which future scholarly research in ethics can be directed.

Mendoza College of Business, University of Notre Dame, Notre Dame, IN 46556-0399, U.S.A. E-mail:atenbrun@nd.edu 